\title{
Selection of suitable excitation and recording conditions for active lock-in thermography with the help of parameter studies
}

G. Schober ${ }^{1}$, C. Kolb ${ }^{1}$, S. Kremling ${ }^{1}$, M. Werner ${ }^{1}$, M. Fink ${ }^{2}$

${ }^{1}$ SKZ - German Plastics Center, Friedrich-Bergius-Ring 22, 97076 Wuerzburg, Germany

2 Julius-Maximilians-Universitaet, Sanderring 2, 97070 Wuerzburg, Germany

g.schober@skz.de

\begin{abstract}
In the field of non-destructive testing (NDT), active lock-in thermography is state of the art. Thereby, the test object is externally excited with different modulated thermal sources. The thermal excitation may be induced optically by lamps, by ultrasonic waves or in case of conductive materials also by eddy currents. Lock-in thermography is frequency-selective, e.g. it responds only to temperature changes at the specific excitation frequency. In theory, the phase image obtained from Fourier analysis shows thermal structures below the surface without influences of inhomogeneities from excitation. However, in reality there are significant influences of several parameters on phase image quality as well as the acquisition and evaluation time and the needed memory capacity. Here, we present a parameter study of relevant optical excitation properties to increase the probability of detection of flaws.
\end{abstract}

Key words: non-destructive testing, active lock-in thermography, phase image, parameter study, probability of detection

\section{State of the art}

In thermography testing, the infrared radiation emitted from an objects surface is detected with high spatial resolution. It serves as a measure of the object's surface temperature. Thermography cameras allow the determination of changes over time and by location. For the use with plastics, applications are usually limited to thin walls in the range of a few millimetres. Thermography is able to measure volumetric flaws as well as to detect flat flaws such as delaminations or the absence of fibre layers in fibre-reinforced composite parts. In active lock-in thermography, the intensity of excitation is usually modulated with the help of lamps [1], ultrasonic waves [2, 3] or eddy currents [4, 5], respectively. It is a frequencyselective method and responds only to temperature changes at the specific excitation frequency. Lock-in thermography is based on heat diffusion into a component which can be described generally by:

$0=\frac{\partial T(\vec{z}, t)}{\partial t}-\alpha \cdot \Delta u(\vec{z}, t)$

$T$ is the temperature at the point $\vec{z}$ and the time $t$ and $\alpha$ is the thermal diffusivity. Assuming the heat diffusion only in the direction of the maximum temperature gradient with a continuous thermal diffusivity, equation (1) is simplified to:

$0=\frac{\partial T(z, t)}{\partial t}-\alpha \cdot \frac{\partial^{2} T(z, t)}{\partial z^{2}}$

The temperature modulation, which is detected from the components surface, has the properties of a damped wave. Assuming a steady local and temporal behaviour a solution for the diffusion equation can be described by:

$T(z, t)=T_{0} \cdot e^{-\frac{z}{\mu}} \cdot e^{i\left(2 \pi f t-\frac{z}{\mu}\right)}$

Here, $f$ describes the so-called lock-in frequency and $\mu$ the thermal penetration depth. The latter is calculated to:

$\mu=\sqrt{\frac{\alpha}{\pi f}}$

Therefore the penetration depth is frequencydependent: the lower the excitation frequency, the greater the depth of penetration and thus the time required for measurement. In theory, phase images obtained from Fourier analysis show structural information below the surface without any influences of the excitation amplitude. However, in reality there are 
significant influences of several parameters on phase image quality as well as on the acquisition and evaluation time and the needed memory capacity, respectively. Quantification studies of these parameters are time and cost consuming but necessary to ensure optimal excitation parameters for each application.

\section{Processing Setup}

Within this work we have studied the influence of varying parameters on a carbon-fiber reinforced plastic component (carbon fibers embedded in an epoxy resin matrix) with cylindrical milling grooves of different sizes and depths. The diameters of the holes are $4.5 \mathrm{~mm}$, $6.0 \mathrm{~mm}, 7.5 \mathrm{~mm}, 9.0 \mathrm{~mm}$ and $10.5 \mathrm{~mm}$ with distances to the sample surface of $0.9 \mathrm{~mm}$, $1.4 \mathrm{~mm}, 1.9 \mathrm{~mm}, 2.4 \mathrm{~mm}, 2.9 \mathrm{~mm}, 3.4 \mathrm{~mm}$ and $3.9 \mathrm{~mm}$. Thermograms for the detection of defects were acquired with a microbolometer infrared camera Jenoptik, VarioCam hr. The technical specifications of the camera are listed in table 1. For excitation in reflection mode four halogen lamps with a total power of $2000 \mathrm{~W}$ in a distance of $30 \mathrm{~cm}$ were used (see fig. 1).

Tab. 1: Technical specifications of the used thermography camera

\begin{tabular}{|l|l|}
\hline Spectral range & $7.5-14.0 \mu \mathrm{m}$ \\
\hline Thermal resolution $\left(30^{\circ} \mathrm{C}\right)$ & $0.05 \mathrm{~K}$ \\
\hline Measuring accuracy & $\begin{array}{l} \pm 1.5 \mathrm{~K} \\
\left(0-100{ }^{\circ} \mathrm{C}\right)\end{array}$ \\
\hline $\begin{array}{l}\text { Temperature } \\
\text { measurement range }\end{array}$ & $-40-1,200^{\circ} \mathrm{C}$ \\
\hline Geometrical Resolution & $\begin{array}{l}640 \times 480 \\
\text { pixels }\end{array}$ \\
\hline Detector type & microbolometer \\
\hline Frame rate & $50 \mathrm{~Hz}$ \\
\hline Focal distance of the lens & $50 \mathrm{~mm}$ \\
\hline Instantaneous field of view & $0.5 \mathrm{mrad}$ \\
\hline
\end{tabular}

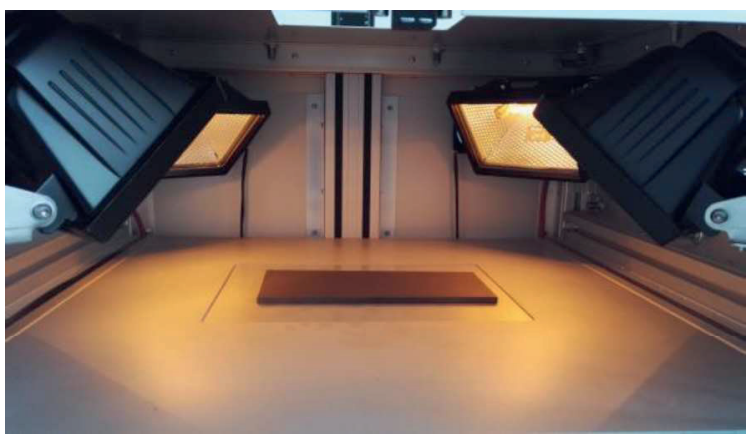

Fig. 1 Photo of a thermography system of four halogen spotlights and a test sample.

\section{Analysis}

Due to the periodic excitation of the surface, a thermal wave is induced and propagates through the part. At interfaces such as homogeneities the thermal wave is reflected back to the surface. This causes a detectable periodic change of surface temperature. Amplitude and the phase images can be calculated by pixel-discrete Fourier analysis. To quantify the presentability of a defect the signalto-noise ratio $(S N R)$ is calculated by the difference of the mean phase values $\varphi_{1}$ and $\varphi_{2}$ and their standard deviations $\sigma_{1}$ and $\sigma_{2}$ of an area with (1) and without (2) a defect beneath the sample surface:

$S N R=\frac{\varphi_{1}-\varphi_{2}}{\sigma_{1}-\sigma_{2}}$

\section{Experiment}

In the following studies milling grooves with a diameter of $10.5 \mathrm{~mm}$ and a distance to the sample's surface of $0.9 \mathrm{~mm}$ (P1) and $1.4 \mathrm{~mm}$ (P2) were considered. In addition, the total averaged $S N R$ for all areas with a defect was investigated, independent whether they are visible or not. For all used frequencies there is an increasing asymptotic correlation of the measuring time and the SNR. The same correlation was observed in case of the averaged SNR for all areas with and without a defect. This is caused by a decreasing standard deviation of all pixels in general and corresponds to Square Root of $\mathrm{N}$ Law. However, the slope for lower excitation frequencies (solid lines) is greater than for higher frequencies (dashed lines, fig. 2). The time, the sample needs to reach a thermal equilibrium and thus a constant local and temporal state is greater in case of a lower frequency. 


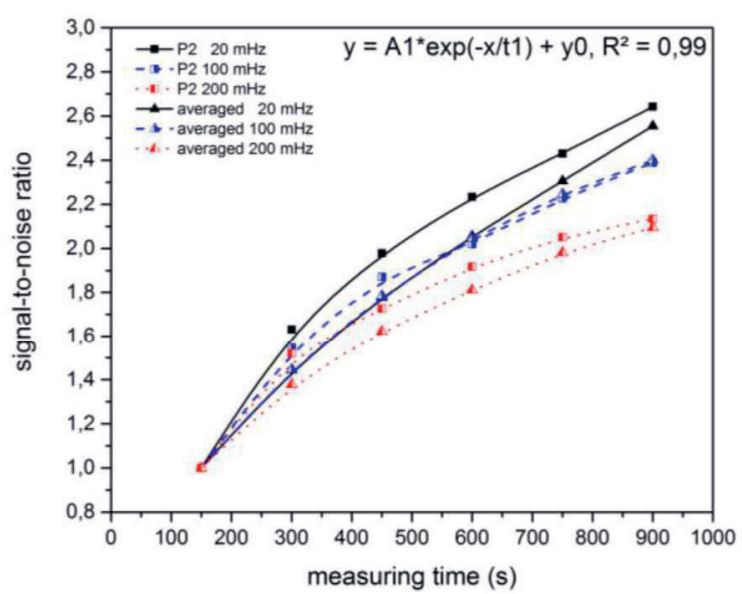

Fig. 2 SNR as a function of the measurement time for three different excitation frequencies of $20 \mathrm{mHz}$, $100 \mathrm{mHz}$ and $200 \mathrm{mHz}$, for $\mathrm{P} 2$ and the averaged SNR of all defects.

In the next step for both defected areas P1 and P2 the influence of the excitation energy was studied in case of an excitation frequency of $20 \mathrm{mHz}$. Therefore the power of the used halogen lamps was reduced from $1.200 \mathrm{~W}$ over $900 \mathrm{~W}$ to $600 \mathrm{~W}$. In addition the number of evaluated periods, which is proportional to the measurement time, was varied as before. The results are shown in Figure 3.

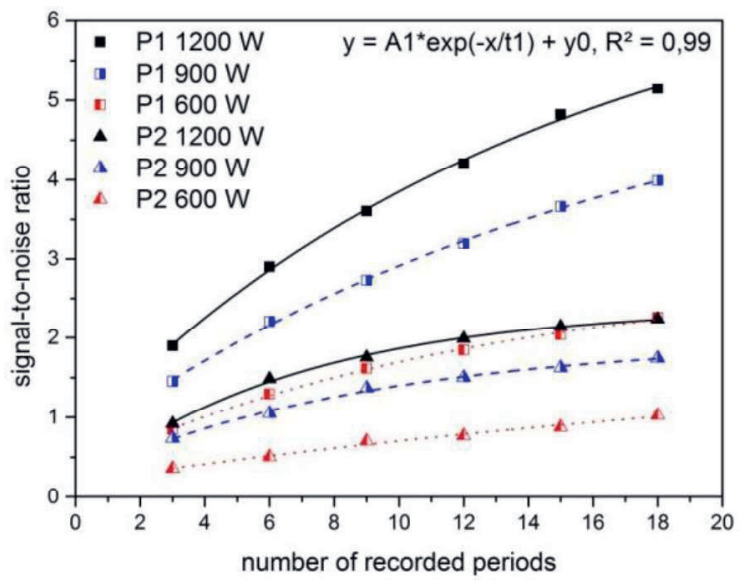

Fig. 3 Correlation of the SNR and excitation power as well as the number of recorded periods.

There is the same dependence as before of the $S N R$ from the number of evaluated periods. Furthermore, a higher excitation power results in a higher SNR. According to a lot of publications the main advantage of phase images is the independence of the excitation amplitude. The phase differences of a defected and defect-free area do not depend on the amplitude and thus the excitation power, but there is a strong correlation to the standard deviations. An increasing excitation power leads to a significant decrease of thermal noise depending on the infrared camera. According to eq. (5) a decreasing standard deviations result in an increasing SNR. In addition the improvement of $S N R$ caused by a higher excitation power depends also on the number of recorded periods. The improvement of SNR caused by a higher excitation power is greater for a larger number of recorded periods than for a small one. As a conclusion, achieving high SNR for good presentability of defects, the excitation power should be chosen as high as possible. There, the thermal stability of the sample has to be taken into account.

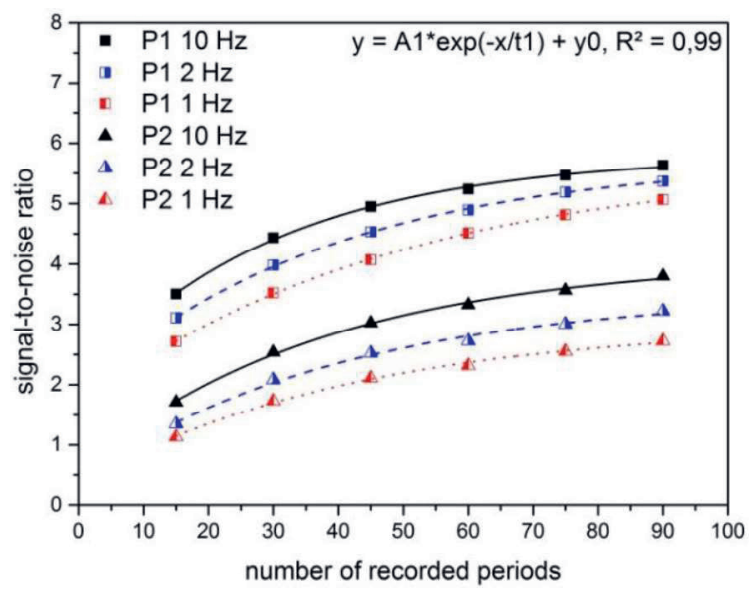

Fig. 4 SNR in dependence of the measuring time for three different recording frequencies of $1 \mathrm{~Hz}, 2 \mathrm{~Hz}$ and $10 \mathrm{~Hz}$ as well as for different locations $P 1$ and $P 2$ in case of an excitation frequency of $100 \mathrm{mHz}$. An asymptotic behaviour is visible for all SNR, not depending on the recording frequency or number of recorded periods.

Figure 4 shows the results of the influence of recording frequency on the SNR of measurements with an excitation frequency of $100 \mathrm{mHz}$. Again, there is an asymptotic increasing but the difference between recording frequencies of $1 \mathrm{~Hz}$ to $10 \mathrm{~Hz}$ is for $\mathrm{P} 1$ much smaller than the difference caused by a varying excitation power between $600 \mathrm{~W}$ and $1.200 \mathrm{~W}$ and for P2 similar. Furthermore the slope almost doesn't depend on the recording frequency or the depth of the defect. In contrast the difference in SNR from $1 \mathrm{~Hz}$ to $2 \mathrm{~Hz}$ seems to be equal as from $2 \mathrm{~Hz}$ to $10 \mathrm{~Hz}$. However, with an increasing recording frequency the amount of data increases significantly. This is the reason why a higher recording frequency should be only chosen if the amount of data is not relevant or the improvement of SNR caused by optimal excitation power parameters is still not sufficient. 


\section{Conclusion}

As a result, the higher the amplitude of the excitation the higher is the SNR of flaws in general. In practical use this correlation is only limited by the maximum permissible surface temperature of the part. To avoid heat damages caused by too high thermal stresses, the thermal stability of the sample must be taken into account very carefully. If the $S N R$ is still not sufficient for the presentability of the defects, the recording duration and recording frequency should be increased. The higher the recording frequency and recording duration the higher is the contrast of flaws. In addition the influence of the recording duration is especially for low excitation frequencies much greater than the influence of the recording frequency. If the needed time for NDT is not relevant, a greater recording duration should be favored. If the SNR is still not sufficient or the measuring time is limited by parameters as the cycle time of a process, the recording frequency should be increased too. Of course, standards in signal processing like Nyquist-Shannon sampling theorem should be considered. Considering the progress of recent years with the focus on miniaturization of the required test hardware and increasing of geometric resolution these results in an enormous amount of data. As a consequence, recording frequency and duration should be chosen as high as necessary and as low as possible.

In summary it can be clearly seen that for every application a careful consideration of the excitation parameters and data recording settings are recommended. This helps the user to decrease the amount of data, the maximum surface temperature during the testing time and increase the contrast of flaws.

\section{Acknowledgment}

The IGF proposal project $18166 \mathrm{~N}$ by the research association "Fördergemeinschaft für das SKZ" was funded through the AiF within the scope of the program for the Promotion of Cooperative Industrial Research and Development (IGF) by the Federal Ministry for Economic Affairs and Energy due to a decree by the German Federal Parliament. We would like to thank them for their financial support. Furthermore we would like to thank the members of the project-related committee for the discussions.

\section{References}

[1] G. Busse, D. Wu, W. Karpen, Thermal wave imaging with phase sensitive modulated thermography, Journal of Applied Physics 71/1992, p. 3962-3965

[2] R. B. Mignogna et al., Thermographic Investigations of High-Power Ultrasound Heating in Materials, Ultrasonics 7/1981, p. 159-163

[3] G. Fischer, Vibrothermographie - ein zerstörungsfreies Verfahren zur Verformungsanalyse und Schadensdetektion bei Kunststoffteilen. VDI Berichte 631, 1987, p. $153-62$

[4] J. Schlichting, Integrale Verfahren der aktiven Infrarotthermografie, dissertation, university of Berlin, 2012

[5] G. Riegert, Induktions-Lockin-Thermografie - ein neues Verfahren zur zerstörungsfreien Prüfung, dissertation, university of Stuttgart 